\title{
PSICOLOGIA \\ ARGUMENTO \\ doi: http://dx.doi.org/10.7213/psicolargum.36.91.AO02 \\ Atuação do Psicólogo no recrutamento e na seleção de pessoas sob a ótica da Análise do Comportamento
}

ISSN 01037013

\section{Psychologist's performance in the recruitment and selection of people from the Behaviourism perspective}

\author{
Pedro Guilherme Basso Machado[a] \\ Paulo Cesar Porto-Martins ${ }^{[\mathrm{b}]}$ \\ Taiana Bueno de Melo Negrelli [c] \\ Laynara de Almeida [d] \\ [a] Docente UniBrasil, pgbmachado@hotmail.com \\ [b] Docente PUCPR, paulocpmar@pucpr.br \\ [c] Acadêmica UniBrasil, taiana.negrelli@gmail.com \\ [d] Acadêmica UniBrasil, laynaradealmeida@gmail.com
}

\section{Resumo}

O presente artigo teve como objetivo descrever a atuação do Psicólogo frente ao processo de recrutamento e seleção (RS) de pessoas, sob a ótica da análise do comportamento. Trata-se de uma revisão de literatura, descritiva, com análise qualitativa. O Psicólogo, ao atuar no recrutamento e na seleção de pessoas, pode contribuir com seus conhecimentos, tanto para a estruturação do processo quanto para a análise dos candidatos e identificação dos perfis comportamentais e técnicos dos profissionais a serem contratados. Para uma contratação assertiva e um processo de seleção eficaz, são estruturadas etapas que envolvem ações do Psicólogo no RS de candidatos, como a aplicação de testes, dinâmicas de grupo e entrevista. O diferencial do Psicólogo está em contribuir com a organização com seus conhecimentos sobre o comportamento humano, além de trazer um olhar humanizado. A descrição e compreensão sobre a atuação do Psicólogo no RS de 
pessoas pode contribuir, entre outros aspectos, para a otimização de processos em prol dos trabalhadores e das empresas. Cabe destacar que algumas das limitações do presente estudo foram em relação à escassez de literatura, nas fontes pesquisadas, sobre a psicologia e o RS, ainda mais quando se trata da perspectiva da Análise do Comportamento. Sugere-se, para futuros estudos, o aprofundamento dos eixos temáticos da presente investigação. Entretanto, destaca-se que o objetivo do presente artigo foi atingido, uma vez que se realizou a descrição sobre a atuação do Psicólogo organizacional frente ao recrutamento e à seleção de pessoas sob a ótica da Análise do Comportamento.

Palavras-chave: Psicólogo organizacional, psicologia organizacional, organizações, recrutamento e seleção, análise do comportamento

\begin{abstract}
The present article aimed to describe the performance of the Psychologist in relation to the recruitment and selection (RS) process from the perspective of the behaviourism. It's a literature review, descriptive, with qualitative analysis. The Psychologist in the recruitment and selection of people can contribute through its knowledge, both for the structuring of the process and for the analysis of the candidates and identification of the behavioural and technical profiles of the professionals to be hired. For an assertive engagement and an effective selection process, steps are structured which involves actions, of the Psychologist in the RS of candidates, such as the application of tests, group dynamics and interview. The differential of the Psychologist is to contribute to the organization through its knowledge about human behaviour, also to bring a humanized look. The description and understanding about the performance of the Psychologist in RS of people can contribute, among other aspects, to the optimization of processes in favour of the workers and companies. It's highlighted that some limitations of the present study were related to the scarcity of literature, in the searched sources, on psychology and RS, even more when it comes to the Behavioural perspective. It's suggested for future studies the deepening of the thematic axes of the present investigation. However, it's highlighted that the objective of this article was reached, once the description of the performance of the Organizational Psychologist in relation to the recruitment and selection of people from the perspective of the Behaviourism was carried out.
\end{abstract}

Keywords: Organizational psychologist, organizational psychology, organizations, recruitment and selection, behaviourism

\title{
Introdução
}

A Psicologia é a ciência e a profissão que possibilita a atuação do profissional Psicólogo em áreas diversificadas de trabalho, dentre elas, a organizacional, foco do presente estudo. Antes mesmo de ser regulamentada no Brasil pela Lei $n$. 4.119, de 27 de agosto de 1962 (Brasil, 1962), a Psicologia ganhou espaço para além do contexto clínico. No início do século XX, segundo Merlo e Silva (2007), os Psicólogos passaram a ser convidados a contribuir com o ambiente organizacional devido ao seu conhecimento sobre comportamento humano, como prevê-lo e como controlá-lo. O foco da atuação do Psicólogo no contexto organizacional era direcionado aos problemas de recrutamento e seleção de pessoas com o objetivo de encontrar o melhor trabalhador para cada função e inclusive sua atuação foi eficaz nesse contexto, pois trouxe, por consequência, a possibilidade de tal 
profissional estender sua contribuição ao setor de Recursos Humanos e para demais áreas dentro das organizações (Zanelli, 1994).

A área de Recursos Humanos, inicialmente denominada "Administração de Pessoal", é o setor responsável por determinadas demandas que envolvem os colaboradores de uma organização, como seleção, contratação, remuneração e benefícios, treinamento, saúde do trabalhador e comunicação entre empresa e colaboradores (Chiavenato, 2010). O desenvolvimento da área teve início em meados do século XX, com a modernização das indústrias e as transformações no desenvolvimento econômico e tecnológico. Tal setor foi ganhando espaço com a influência direta das movimentações das relações sindicais e trabalhistas da época, que trouxeram um comportamento mais ativo dos trabalhadores, os quais reivindicavam maiores benefícios e melhor qualidade no dinâmico ambiente laboral (Zanelli, 1994).

Destaca-se que as empresas vivem em constante processo de mudança e o setor de Recursos Humanos é uma área estratégica nas organizações, uma vez que a atuação requer cautela e conhecimento (Espindola \& Oliveira, 2009). Atualmente, dentre as principais funções do setor de Recursos Humanos, está o processo de recrutamento e seleção de pessoas, que pode ser conduzido por profissionais de diversas áreas e formações acadêmicas, inclusive pelo Psicólogo. Recrutamento é o conjunto de procedimentos que visa atrair pessoas potencialmente qualificadas para ocupar um cargo dentro da organização. Após atrair candidatos em potencial, inicia-se o processo de seleção, a fim de identificar o melhor perfil, aquele que possui as competências técnicas e comportamentais ideais para desempenhar uma determinada função em um ambiente organizacional específico (Bohlander, Snell \& Shermam, 2003; Chiavenato, 2006).

Nesse contexto, entre os diferentes referenciais teóricos que o Psicólogo Organizacional pode utilizar na sua atuação está a Análise do Comportamento (AC), abordagem que merece destaque no cenário nacional, uma vez que o Brasil "[...] concentra a maior comunidade de analistas do comportamento fora dos Estados Unidos" (Strapasson, Zuge \& Cruz, 2017, p. 97). Essa abordagem direciona estudos para a compreensão e o controle do comportamento por meio da sua interação com o ambiente, a fim de analisar quais são as causas desse comportamento para então compreender suas funções (Moreira \& Medeiros, 2007) e assim aumentar a probabilidade de controlá-los (Skinner, 2003).

Para Skinner (2003), comportamento é a interação entre organismo e ambiente, que forma um fenômeno singular de inter-relação, já que o comportamento promove modificações no ambiente e ao mesmo tempo é influenciado por elas, ou seja, o comportamento é determinado pelas relações com o ambiente (Dittrich, 2012). Entende-se por ambiente qualquer evento, privado ou externo, capaz de afetar o organismo (Skinner, 2003), isto é, a junção de variáveis entre o mundo físico, social e história de vida (Moreira \& Medeiros, 2007). Segundo Todorov (2007, p. 59), "Não há sentido em uma descrição de comportamento sem referência ao ambiente, como não há sentido, para a Psicologia, em uma descrição do ambiente apenas". Dentre os diversos tipos de ambiente existentes, há o organizacional, contexto do presente estudo, que pode ser definido como o 
conjunto de fatores internos e externos que influenciam nos processos e no desempenho de uma organização (Ferreira \& Santos, 2013). Sendo assim, o comportamento dos colaboradores opera mudanças no ambiente organizacional, assim como seu próprio comportamento pode ser modificado conforme as contingências presentes nesse ambiente.

Para a Análise do Comportamento, contingência refere-se a todos os componentes das relações comportamentais, as quais possuem uma relação de dependência entre si (Dias, Souza \& Teixeira-Júnior, 2005). É um instrumento conceitual que considera interação entre estrutura do ambiente e estrutura do comportamento (Todorov, 2012). Expressa relações entre o organismo e seu ambiente, que modelam o comportamento devido as suas consequências (Dias et al., 2005; Moreira \& Medeiros, 2007).

Dentre os tipos de consequências geradas pelo comportamento está o reforço, o qual aumenta a probabilidade de este voltar a ocorrer, pela adição de estímulo reforçador ou remoção de estímulo aversivo (Baum, 2006; Moreira \& Medeiros, 2007). Ou seja, o ambiente passa a controlar o comportamento a partir da relação que estabelece com as consequências reforçadoras (Azevedo \& Todorov, 2016).

Cabe destacar que a organização é o local no qual muitas pessoas passam maior parte do tempo e essas pessoas têm a força necessária para movê-la ou ser uma grande barreira (Espíndole \& Oliveira, 2009). Por isso, a compreensão das características comportamentais dos candidatos faz-se essencial na identificação do perfil ideal durante o processo de seleção de pessoas, pois somente os aspectos técnicos não se fazem suficientes para atender à demanda das organizações. Portanto, objetiva-se, por meio dessa compreensão, otimizar os processos de recrutamento e seleção nas organizações (Franco, Miguel \& Santos, 2003).

O estudo sobre o trabalho do Psicólogo frente ao recrutamento e à seleção de pessoas deve visar a identificação das possíveis contribuições que esse profissional, por meio de seus conhecimentos, de suas técnicas e de alguns instrumentos, poderá trazer para a efetividade dos processos de contratação e, consequentemente, para o ambiente organizacional, para a produtividade e para o crescimento de uma organização (Ferreira \& Santos, 2013). Por isso, a importância do presente estudo, que objetivou descrever a atuação do Psicólogo organizacional frente ao processo de recrutamento e seleção de pessoas sob a ótica da Análise do Comportamento. Destaca-se, ainda, o defendido por Strapasson et al. (2017) sobre a necessidade de publicações que utilizam fundamentos da análise do comportamento, em especial nesse contexto.

\section{Metodologia}

Foi realizada revisão de literatura, descritiva, com análise qualitativa e utilizado o método da análise de conteúdo preconizado por Bardin (1977), que é um conjunto de técnicas utilizadas para analisar comunicações por procedimentos sistemáticos e objetivos. 
Como fontes de pesquisa, buscaram-se obras disponíveis em acervo físico, numa biblioteca de uma Instituição de Ensino Superior, assim como realizaram-se consultas on-line no site do Conselho Federal de Psicologia, na base de dados EBSCO e por meio do mecanismo de busca Google Acadêmico, que fornece acesso a distintas bases de dados, como o SCIELO (Scientific Electronic Library Online), PEPSIC (Periódicos Eletrônicos de Psicologia), entre outras fontes. Foram selecionados, como referência, livros e artigos científicos relacionados à temática do presente estudo.

Como critério de busca, utilizaram-se as seguintes palavras-chave: "Psicologia Organizacional", "Psicólogo e Recrutamento e Seleção", "Recrutamento e Seleção de Pessoas", "Recrutamento e Seleção e Análise do Comportamento", "Psicologia Organizacional e Análise do Comportamento", "Análise do Comportamento".

Já para os critérios de inclusão, utilizaram-se obras consideradas funcionais para descrever as características da atuação do psicólogo nos processos de recrutamento e seleção que possibilitem discorrer sob a ótica da análise do comportamento. Como critério de exclusão, suprimiram-se os trabalhos em língua estrangeira, uma vez que o foco foi discorrer sobre a realidade do recrutamento e da seleção sob a ótica da análise comportamental pertinente à realidade brasileira.

\section{Resultados}

Após o crivo dos critérios de inclusão e exclusão, selecionaram-se 18 artigos científicos, 11 livros e 1 resolução que, mediante a análise de conteúdo, geraram quatro categorias:

- atuação do Psicólogo no recrutamento e na seleção;

- recrutamento;

- seleção (testes, dinâmicas de grupo, entrevista);

- contribuições do Psicólogo para o processo de recrutamento e seleção.

\section{Atuação do Psicólogo no recrutamento e seleção}

Inicialmente, a área de recursos humanos dedicava-se a registrar faltas e atrasos com vistas ao desconto salarial, mas com o avançar do tempo, começou a atuar de maneira estratégica ao gerir processos desde a atração de candidatos para as vagas ofertadas pela organização, até o desenvolvimento e a manutenção de alta performance (Paiva et al., 2017).

Dentro do contexto de recrutamento e seleção, o Psicólogo tem papel fundamental para o desenvolvimento da organização, pois os objetivos organizacionais somente são atingidos por meio das pessoas que a compõem (Arieira \& Guimarães, 2005). Cada vez mais globalizadas, tecnológicas e competitivas, as organizações buscam pessoas capacitadas tecnicamente e que possuam um perfil comportamental alinhado a sua visão, a sua missão e aos seus valores, visando atingir melhores resultados, que promovam crescimento organizacional (Franco et al., 2003). 
O conhecimento do Psicólogo em relação à organização em que está inserido influencia diretamente em sua atuação. Para fundamentar suas ações e estratégias, é primordial a compreensão do Psicólogo sobre a história da organização, suas características, o tipo de gestão, as condições organizacionais vigentes, os serviços e ou produtos que fornece, dentre outros aspectos. Tais informações fornecerão subsídios para que o Psicólogo elabore o processo de recrutamento e seleção alinhado à cultura e ao ambiente organizacional (Chaves \& Nunes, 2010).

Cabe destacar que as condições organizacionais e o incentivo comportamental existente por parte da gestão podem limitar ou expandir as possibilidades da atuação dos profissionais que a compõem (Chaves \& Nunes, 2010). Para a elaboração de um processo de recrutamento e seleção, o Psicólogo depende do investimento motivacional, financeiro, entre outros recursos que a organização dispõe para a execução do processo e para o desenvolvimento da área. Conforme as condições expostas, o Psicólogo poderá planejar o processo visando, por exemplo, a redução de custos ou então o investimento em ferramentas que podem auxiliar no êxito do trabalho (Ferreira \& Santos, 2013).

Para o planejamento do processo de recrutamento e seleção que será realizado, o ideal é que seja feita uma análise da descrição do cargo a ser trabalhado, a fim de avaliar principalmente as competências técnicas e comportamentais descritas para ele. Esses dados servirão como base para a definição das estratégias de recrutamento e para a estruturação das etapas de seleção (Chiavenato, 2010; Coradini \& Murini, 2009), segundo as exigências impostas pela organização (D'Ávila, Régis \& Oliveira, 2010; Hora, Lima, Santos, Oliveira \& Souza, 2017).

A descrição de cargos é de suma importância para toda e qualquer organização, independentemente de sua estrutura e da diversidade de cargos que possui. É um dos alicerces essenciais para todas as ações do setor de RH, sendo um antecedente fundamental para o recrutamento e seleção, treinamento e desenvolvimento, estruturação de plano de carreira, administração de salários, avaliação de desempenho, entre outras (Arieira \& Guimarães, 2005; Bohlander et al., 2003; Chiavenato, 2010).

Conforme Tadaiesky (2008), ao analisar a descrição do cargo, o Psicólogo poderá identificar as possíveis relações de contingências e comportamentos que estão associados às tarefas mais importantes do cargo, levando em conta as variáveis ambientais, como ambiente organizacional, estrutura da empresa, divisão do setor, tipo de gestão, os quais exercem controle sobre os comportamentos do funcionário, sendo então possível identificar as principais competências técnicas e comportamentais dos candidatos que deverão ser atraídos para participar do processo e aumentar sua probabilidade de êxito.

A seguir, são apontadas as características específicas dos processos de recrutamento.

\section{Recrutamento}


O objetivo de recrutar consiste em atrair candidatos para a realização da seleção. Geralmente a organização convida profissionais para participar do processo de seleção para um cargo específico (Bohlander et al., 2003; Chiavenato, 2006).

O papel do Psicólogo nessa etapa requer estratégia para que sejam recrutados profissionais que estejam alinhados às exigências do cargo. Por isso, a decisão dos locais de divulgação das oportunidades bem como as informações divulgadas são de extrema importância para aumentar a probabilidade de êxito do processo e evitar a atração de perfis que não possuem os requisitos mínimos para o exercício da função (Silva \& Silva, 2013).

Dentre as principais decisões a serem tomadas nessa etapa do processo, está o tipo de recrutamento que será realizado: interno, externo ou misto (D'Ávila et al., 2010; Lacombe \& Heilborn, 2003). O processo de recrutamento interno refere-se à divulgação da vaga em aberto para os próprios colaboradores, que terão a oportunidade de participar do processo de seleção visando uma promoção ou o remanejamento para outro setor almejado. O recrutamento externo busca profissionais que estão fora da organização, ou seja, que se encontram disponíveis no mercado de trabalho, empregados ou não. Já o recrutamento misto é a realização do processo interno e externo concomitantemente, sendo esse o tipo de recrutamento mais utilizado pelas organizações de grande porte (Chiavenato, 2006). Vale ressaltar que os tipos de recrutamento podem possuir prejuízos e benefícios em seu processo - menor ou maior custo, tempo, adaptação a nova função ou ao clima da empresa - e cabe ao Psicólogo analisar as relações de contingências ambientais e a estruturação da equipe em questão, para definir, em conjunto com os responsáveis, a melhor estratégia para cada processo especificamente (Coradini; Murini, 2009; Franco et al., 2003).

O recrutamento bem planejado resulta na otimização da etapa de seleção. Conforme mencionado, a junção de uma boa descrição da vaga com a divulgação em locais e meios de comunicação pertinentes ao cargo aumentam a probabilidade de atrair profissionais alinhados ao que a organização busca, agilizando, assim, as etapas seguintes do processo de acordo com os objetivos organizacionais (Silva \& Silva, 2013).

A primeira ação após a atração dos candidatos é a triagem de currículos dos profissionais interessados em participar do processo. Nessa ação, denominada pré-seleção, é realizada uma análise técnica, na qual serão eliminados os currículos que não possuem os requisitos fundamentais exigidos, como, por exemplo, experiência anterior na função e formação educacional (Tadaiesky, 2008). Em seguida, a realização de uma análise mais detalhada dos currículos préselecionados guiará o Psicólogo na convocação dos candidatos para o processo seletivo (Coradini \& Murini, 2009).

Cabe destacar que somente o currículo não se faz suficiente para uma contratação, porém, a boa estruturação e o detalhamento dele é de extrema importância para que o Psicólogo possa convocar, para a etapa de seleção, os profissionais que possuem características compatíveis com as exigências da vaga (Silva \& Silva, 2013). 


\section{Seleção}

Realizada a atração dos candidatos e a pré-seleção dos currículos, inicia-se o processo de seleção efetivamente, que consiste no conjunto de atividades e técnicas que forneçam subsídios para a escolha do profissional que irá ocupar o cargo disponível (Chiavenato, 2010). O Psicólogo Organizacional poderá estruturar as etapas de seleção e atrelar seus conhecimentos às contingências do ambiente organizacional, podendo utilizar instrumentos que venham a contribuir para a investigação do repertório comportamental dos candidatos (D'Ávila et al., 2010; Lacombe \& Heilborn, 2003; Tadaiesky, 2008).

O objetivo dessa etapa é contratar o profissional que possui competências técnicas e comportamentais essenciais para o exercício da função, as quais estejam alinhadas à cultura e às características da empresa dentre a mão de obra disponível. Segundo Chiavenato (2006) e Coradini e Murini (2009), muitas organizações, devido a sua cultura e a sua estrutura, utilizam-se somente da entrevista como estratégia para o processo de seleção, porém, mesmo sendo considerada a ação mais importante para um processo de seleção, esses autores enfatizam a importância de uma seleção estruturada, com técnicas que forneçam subsídios suficientes para a melhor avaliação dos candidatos. Cabe destacar ainda que a utilização das técnicas, bem como a ordem de realização e aplicação delas, vai depender, dentre outros aspectos, da demanda da organização, dos recursos disponíveis e da forma como o Psicólogo avalia a vaga em questão e estrutura o processo (Arieira \& Guimarães, 2005).

O sucesso do processo de seleção está relacionado ao processo de mapeamento das necessidades organizacionais que pretendem ser supridas com essa nova vaga, assim como utilizar todos os recursos e técnicas possíveis para avaliar o candidato, inclusive mediante investigação do networking do candidato (D'Ávila et al., 2010).

A seguir, são especificadas algumas das atividades que podem servir de apoio ao Psicólogo Organizacional no processo de seleção.

\section{Testes}

A aplicação de testes no processo de seleção é utilizada como ferramenta para dois objetivos principais: mensurar o nível de conhecimento técnico e estimar os comportamentos que os candidatos poderão emitir como resposta às contingências e aos estímulos presentes no ambiente de trabalho (Bohlander, et al., 2003). Os testes mais utilizados nas organizações são os práticos, situacionais e psicológicos (Paiva et al., 2017).

A diversidade de ferramentas utilizadas no processo de seleção vem aumentando por meio da contribuição dos profissionais de diferentes formações que atuam nessa função, na tentativa de trazer mais assertividade ao processo e poder, nessa fase, auxiliar a selecionar os candidatos que possuem requisitos necessários ao preenchimento da vaga (Godoy \& Noronha, 2005). 
Os testes que objetivam a avaliação de conhecimento técnico geralmente são utilizados em cargos que exigem conhecimento específico em determinado assunto, como, por exemplo, conhecimento em Tecnologia da Informação (TI). Tais avaliações podem ser desenvolvidas pelo gestor da área, que possui o conhecimento técnico a ser avaliado e conhece as atividades que o candidato irá exercer em caso de aprovação, por isso, é a pessoa indicada para a elaboração de tal instrumento (Rabaglio, 2013).

$\mathrm{Na}$ tentativa de prever e mensurar os comportamentos/características psicológicas dos candidatos, os testes psicológicos são utilizados devido ao reconhecimento e à legitimidade que frequentemente possuem (Godoy \& Noronha, 2005). Em relação a essas ferramentas, o Conselho Federal de Psicologia (2001) publicou a resolução 025/2001, que define o teste psicológico como uso privativo do Psicólogo, regulamentando sua utilização e comercialização. Contudo, também é possível encontrar referências que apontam a aplicabilidade desses instrumentos no contexto organizacional sem especificar o profissional que conduz esse processo, o que é foco de opiniões divergentes (Franco et al., 2003; Tadaiesky, 2008).

Skinner (2003) aponta que as medidas fornecidas pelos testes permitem a identificação de traços referentes ao repertório comportamental dos indivíduos e que um mesmo padrão de resposta pode ser resultado de diversas funções adaptativas de interações com o ambiente no qual ocorreram durante a história do indivíduo. Ou seja, variáveis da história do indivíduo influenciam em seu comportamento presente em situação de teste. Por isso, os autores Franco et al. (2003) e Tadaiesky (2008) enfatizam também a importância da utilização de técnicas que promovam a manifestação direta do comportamento, como, por exemplo, as dinâmicas de grupo, em que o Psicólogo pode estar em contato com o candidato e observá-lo em ação.

Os testes psicológicos utilizados em conjunto com demais técnicas servem como ferramenta de verificação dos aspectos observados pelo Psicólogo durante o processo e podem legitimar sua análise. Já a junção das demais técnicas utilizadas com os resultados fornecidos pelo teste podem resultar em uma decisão mais assertiva com relação à escolha do profissional a ser contratado (Godoy \& Noronha, 2005).

\section{Dinâmica de Grupo}

Todo comportamento deve ser analisado em relação ao contexto e esta é uma das principais funções da dinâmica de grupo em ambiente de seleção quando se objetiva observar os candidatos em situação semelhante ao dia a dia no trabalho (Bohlander et al., 2003; Tadaiesky, 2008). Dinâmicas de grupo são vivências nas quais um grupo de pessoas executa uma atividade para atingir objetivos específicos, vivenciando e refletindo sobre tal experiência após sua execução (Coradini \& Murini, 2009).

Por meio de manipulações de variáveis ambientais como dramatizações, jogos ou simulações de situações semelhantes às do contexto de trabalho, o 
Psicólogo poderá observar a ação direta dos candidatos, sendo possível identificar a existência de comportamentos específicos que estão presentes no repertório comportamental de cada um e avaliar se eles contribuem ou não para o exercício da função (Coradini \& Murini, 2009; Rabaglio, 2013; Tadaiesky, 2008). Tal observação, somada às demais informações obtidas em outras fases do processo, permite uma análise mais fidedigna com relação ao possível desempenho do candidato no exercício da função (Tadaiesky, 2008).

\section{Entrevista}

A técnica mais utilizada para selecionar candidatos é a entrevista, já que possui maior credibilidade para a tomada de decisão, sendo considerada por muitos autores a etapa mais importante de todo o processo (Coradini \& Murini, 2009; Rabaglio, 2013; Tadaiesky, 2008). É um momento de troca entre o Psicólogo e o candidato, tendo por finalidade investigar sobre as experiências profissionais, características pessoais e conhecimentos do candidato, aprofundando e esclarecendo questões mencionadas no currículo e ou em contatos prévios.

O roteiro elaborado pelo Psicólogo deve direcionar o candidato ao relato dos comportamentos relevantes presentes em seu repertório, para que seja possível identificar as "[...] contingências nas quais estes são emitidos e dos reforços aos quais esse indivíduo é sensível" (Tadaiesky, 2008, p. 133). Na mesma obra, a autora destaca ainda que para diminuir a probabilidade de um relato falso por parte do candidato, o Psicólogo deve conduzir os questionamentos para que este relate seus comportamentos passados e as consequências deles frente às diversas situações em seu ambiente de trabalho, solicitando, em seguida, exemplos de situações hipotéticas em que o candidato se comportaria de maneira diferente para atingir objetivos ideais.

Com base no pressuposto de que o comportamento passado prediz o comportamento futuro, a investigação de eventos passados da história de vida do candidato, mesmo fora do contexto de trabalho, pode ser fundamental na identificação de padrão comportamental e suas contingências de reforçamento. É um excelente método a ser utilizado em entrevistas, especificamente quando o cargo almejado não requer experiência anterior na função, sendo esta uma oportunidade para que o candidato possa demonstrar sua compatibilidade com o cargo (Franco et al., 2003).

As entrevistas podem ser estruturadas, semiestruturadas ou abertas, uma vez que são técnicas que podem ser utilizadas de diferentes formas, como, por exemplo, a entrevista comportamental (investigação de características comportamentais), entrevista situacional (investigação de possíveis comportamentos futuros frente a situações hipotéticas) e entrevista por competência (investigação de habilidades específicas com base no relato de experiências anteriores). Todas possuem sua eficiência e são utilizadas conforme o planejamento do processo feito pelo Psicólogo ao analisar as especificidades da organização e do cargo a ser trabalhado (Rabaglio, 2013). 
Entrevistar é investigar o máximo possível sobre o repertório comportamental do candidato e identificar suas habilidades, conhecimentos, competências e limitações (Bohlander et al., 2003). Conforme mencionado anteriormente, a entrevista possui maior relevância dentre todas as etapas, pois é a única ação indispensável para qualquer processo seletivo, que será base fundamental para contratar ou não o novo colaborador em relação às especificidades do cargo e da organização (Coradini \& Murini, 2009).

\section{Contribuições do Psicólogo para o processo de recrutamento e seleção}

Não só o Psicólogo pode estar à frente do recrutamento e da seleção de pessoas nas organizações, porém, conforme o objetivo do presente estudo, abaixo estão destacadas algumas contribuições que o Psicólogo Organizacional pode trazer a esse contexto.

Atualmente, muitas organizações possuem uma visão ampliada quanto aos requisitos para a contratação de um novo colaborador, entendendo a importância da análise de perfil comportamental como primordial para contratação. Esse fato faz com que o espaço dos Psicólogos no ambiente organizacional, especificamente no recrutamento e na seleção, seja cada vez mais consolidado (Franco et al., 2003; Merlo \& Silva, 2007).

Um dos princípios da Análise do Comportamento é olhar o indivíduo como único (Franco et al., 2003), levando em conta sua história de vida, seu repertório comportamental e suas relações, o que permite ao Psicólogo uma percepção dos candidatos, que vai além das suas experiências como profissional, pois as características comportamentais, além das técnicas, podem interferir nas ações dos profissionais e facilitar a análise de perfil dos candidatos que estão inclinados a suprir ou até mesmo superar as expectativas da organização (Zanelli, 1994).

A Psicologia, além de enxergar o ser humano como um ser dotado de potencialidades e especificidades, compreende o homem como um ser social, o qual se desenvolve a partir das relações que estabelece. O diferencial do Psicólogo está em contribuir com a organização por meio de seus conhecimentos técnicos sobre "[...] os princípios do comportamento, individual e grupal, os fundamentos da aprendizagem de comportamentos, o seu reforço, a motivação e satisfação laboral" (Chaves \& Nunes, 2010, p. 4). O Psicólogo traz para a organização um olhar humanizado, voltado aos indivíduos e aos seus potenciais, a fim de colaborar com a organização de forma construtiva e alinhar a relação e as expectativas entre empresa e futuros colaboradores (Zanelli, 1994).

Cabe destacar ainda a significativa contribuição do Psicólogo quanto às técnicas e às ferramentas, muitas vezes exclusivas, utilizadas individualmente ou em grupo, que enriquecem o processo seletivo e têm forte contribuição em ações e tomadas de decisões quanto a processos organizacionais, em especial ao recrutamento e à seleção de pessoas (Godoy \& Noronha, 2005).

\section{Considerações finais}


A compreensão da atuação do Psicólogo organizacional no recrutamento e na seleção de pessoas pode contribuir para a ampliação do conhecimento de uma relevante área da Psicologia, podendo colaborar para a adaptação e a boa performance dos profissionais que desejam seguir essa área de atuação. Também pode colaborar para a otimização dos processos organizacionais em prol dos trabalhadores e das empresas.

Utilizando conhecimentos e técnicas específicas, é possível que o Psicólogo contribua com maior assertividade ao processo de contratação, por meio da identificação dos candidatos que possuem comportamentos que podem reforçar modificações desejáveis no ambiente organizacional. Porém é pertinente destacar que em todas as etapas do processo de RS, podem haver manipulações de condutas para aumentar a chance de contratação no processo e cabe ao Psicólogo estar atento a esses aspectos (Tadaiesky, 2008).

Cabe destacar ainda que uma das limitações do presente artigo refere-se à literatura encontrada, a qual, em sua maioria, foi composta por autores das áreas de administração e gestão de recursos humanos, que abordam a temática sobre o recrutamento e a seleção de pessoas. Foram encontradas pesquisas da área da Psicologia sobre a atuação do Psicólogo nas organizações de forma generalista, as quais abordam diversas possibilidades de atuação do Psicólogo nesse contexto, porém notou-se escassez, nas fontes pesquisadas, de literatura em relação ao Psicólogo e ao recrutamento e à seleção de pessoas especificamente, ainda mais quando se trata de obras sob a perspectiva da Análise do Comportamento. Esse estudo, pelo próprio escopo, não abordou outras questões relevantes ao recrutamento e à seleção, como, por exemplo, o processo de integração do funcionário, o acompanhamento de adaptação dele ao ambiente organizacional e as resistências individuais e organizacionais que podem ser encontradas no processo.

Sugere-se que sejam realizados futuros estudos na área da Psicologia organizacional com foco na atuação do Psicólogo frente ao recrutamento e à seleção de pessoas com a perspectiva da Análise do Comportamento, tanto no aprofundamento dos eixos temáticos abordados como na investigação de outras possíveis ações do Psicólogo nesse contexto, para melhor compreendê-lo.

Destaca-se que a literatura generalista em Psicologia praticamente ignora as contribuições da Análise do Comportamento, constituindo uma forma de isolamento específica da Análise do Comportamento na literatura nacional. Sugestões são apresentadas a respeito de como diminuir esse isolamento (Strapasson et al., 2017).

Por fim, afirma-se que o objetivo do presente estudo foi atingido, ou seja, foram descritas as possibilidades de atuação do Psicólogo Organizacional frente ao recrutamento e à seleção de pessoas, atuação esta que pode afetar significativamente, entre outros aspectos, o clima da empresa, os custos, a motivação dos antigos e novos funcionários e consequentemente a sua produtividade (Ferreira \& Santos, 2013).

\section{Referências}


Arieira, J. O., \& Guimarães, M. F. (2005). O processo de recrutamento e seleção como uma ferramenta de gestão. Revista Ciências Empresariais da UNIPAR, 6(2), 203-214.

Azevedo, R. M. F., \& Todorov, J. C. (2016). Controle de estímulos e contraste comportamental em uma tarefa de cooperação. Revista Brasileira de Análise do Comportamento, 12(2), 95-105.

Bohlander, G., Snell, S., \& Shermam, A. (2003). Administração de recursos humanos. São Paulo: Pioneira Thomson Learrnig.

Bardin, L. (1977). Análise de conteúdo. Lisboa: Edições 70.

Baum, W. M. (2006). Compreender o Behaviorismo: comportamento, cultura e evolução. Artmed: Porto Alegre.

Brasil. Lei n. 4.119, de 27 de agosto de 1962. (1962, 14 de Dezembro). Dispõe sobre os cursos de formação em psicologia e regulamenta a profissão de psicólogo. Diário Oficial da União. Recuperado de: http://www.planalto.gov.br/ccivil_03/leis/1950-1969/L4119.htm.

Conselho Federal de Psicologia. (2001, 30 de novembro). Resolução 025/2001. Define teste psicológico como método de avalição privativo do psicólogo. Recuperado

de: http://www.crprs.org.br/upload/legislacao/legislacao46.pdf.

Chaves, N. A., \& Nunes, R. V. (2010). O papel do psicólogo na gestão de recursos humanos das organizações. Revista Científica Eletrônica de Psicologia, 14, 1-7.

Chiavenato, I. (2006). Recursos humanos: o capital humano das organizações (8a ed.). São Paulo: Atlas.

Chiavenato, I. (2010). Gestão de pessoas: o novo papel dos recursos humanos nas organizações (3a ed.) Rio de Janeiro: Elsevier.

Coradini, J. R., \& Murini, L. T. (2009). Recrutamento e seleção de pessoal: como agregar talentos à empresa. Ciências Sociais Aplicadas, 5(1), 55-78.

D'Ávila, G. C., Régias, H. P., \& Oliveira, L. M. B. (2010). Redes sociais e indicações para processos de recrutamento e seleção: uma análise pela perspectiva dos candidatos. Revista Psicologia Organizações e Trabalho, 10(1), 65-80.

Dias, M. F., Souza, M. A. O., \& Teixeira-Júnior, R. R. (2005). Vocabulário de Análise do Comportamento. São Paulo: Esetec. 
Dittrich, A. (2012). O conceito de liberdade e suas implicações para a clínica. In: Borges, N. B. \& Cassas, F. A. (orgs.). Clínica analíticocomportamental. Porto Alegre, Artmed. pp.87-94.

Espindola, M. B. \& Oliveira, A. P. V. D. (2009). Análise comportamental: um estudo de como o comportamento organizacional pode influenciar o clima organizacional de uma indústria. Revista Digital FAPAM, 1(1), 179200.

Ferreira, S. F., \& Santos, F, S. (2013). A importância do recrutamento e seleção de pessoas em uma empresa de pequeno porte do setor de móveis. Revista Eletrônica Diálogos Acadêmicos, 4(1), 46-56.

Franco, R. N. A., Miguel, C. F., \& Santos, J. G. W. (2003). Seleção de pessoal: considerações preliminares sobre a perspectiva Behaviorista Radical. Psicologia: Reflexão e Crítica, 16(2), 235-243.

Godoy, S. L., \& Noronha, A. P. P. (2005). Instrumentos psicológicos utilizados em seleção profissional. Revista do Departamento de Psicologia - UFF, 17(1), 139-159.

Hora, D., Lima, J., Santos, K., Oliveira, N., \& Souza, L. M. S. M. (2017). A Inserção do Jovem no Mercado de Trabalho. Augusto Guzzo Revista Acadêmica, 1(19), 413-425.

Lacombe, F. J. M., \& Heilborn, G. L. J. (2003). Administração: princípios e tendências. São Paulo: Saraiva.

Merlo, A. R. C., \& Silva, P. C. (2007). Prazer e sofrimento de psicólogos no trabalho em empresas privadas. Psicologia Ciência e Profissão, 27(1), 132-147.

Moreira, M. B., \& Medeiros, C. A. (2007). Princípios básicos de Análise do Comportamento. Porto Alegre: Artmed.

Paiva, L. E. B., Lima, T. C. B., Oliveira, T. S., \& Pitombeira, S. S. R. (2017). Percepção da influência das políticas e práticas de recursos humanos na satisfação com 0 trabalho. Revista Pensamento Contemporâneo em Administração, 11(1), 55-69.

Rabaglio, M. O. (2013). Gestão por competências: ferramentas para atração e captação de talentos (2a ed.). Rio de Janeiro: Qualitymark.

Silva, R. P., \& Silva, S. C. (2013). Recrutamento e Seleção de pessoas. Revista InterAtividade, 1(1), 02-10.

Skinner, B. F. (2003). Ciência e comportamento humano (11a ed.). São Paulo: Martins Fontes. 
Strapasson, B. A., Zuge, P. R., \& Cruz, R. N. (2017). O isolamento da Análise do Comportamento no Brasil: uma análise bibliométrica. Revista Brasileira de Terapia Comportamental e Cognitiva, 19(1), 94-114.

Tadaiesky, L. T. (2008). Métodos de seleção de pessoal: discussões preliminares sob o enfoque do behaviorismo radical. Psicologia Ciência e Profissão, 28(1), 122-137.

Todorov, J. C. (2007). A psicologia como o estudo de interações. Psicologia: Teoria e Pesquisa, 23, 57-61.

Todorov, J. C. (2012). Sobre uma definição de comportamento. Perspectivas, 3(1), 32-37.

Zanelli, J. C. (1994). O Psicólogo nas organizações de trabalho: formação e atividades profissionais. Florianópolis: Paralelo 27. 